\title{
Transient liquid phase bonding of pairings of parent superalloy material with different composition and grain structure
}

\author{
S. Steuer ${ }^{1, a}$, S. Piegert ${ }^{2, b}$, M. Frommherz ${ }^{3, c}$, R. F. Singer ${ }^{1}$, A. Scholz ${ }^{3}$
}

${ }^{1}$ Institute of Science and Technology of Metals, Department of Materials Science and Engineering, University of Erlangen, Martensstr. 5, 91058 Erlangen, Germany

${ }^{2}$ Siemens AG, E F PR GT M\&T MPD 3, Huttenstraße 12, 10553 Berlin, Germany

${ }^{3}$ Institut für Werkstoffkunde, Technische Universität Darmstadt, Grafenstr. 2, 64283 Darmstadt, Germany

a Susanne.Steuer@ww.uni-erlangen.de, b sebastian.piegert@siemens.com,

c frommherz@mpa-ifw.tu-darmstadt.de

Key words: TLP bonding, superalloy, microstructure, mechanical properties

\begin{abstract}
Joining of different nickel-base superalloys could simplify the manufacturing of turbine blades. The used technique of choice is transient liquid phase bonding, which is an established repair technology for high temperature components. Two nickel-base superalloys with distinct composition and grain structure are bonded and the joints are analysed regarding the microstructure. To quantify the mechanical properties of these joints, tensile and short term creep rupture tests were performed at room and elevated temperatures.
\end{abstract}

\section{Introduction}

The interest in joining nickel-base superalloys has increased through the last years, because it shows the potential to simplify manufacturing of blades and vanes of modern gas turbines. As the size of these blades increases, so does the probability of casting defects. This could be avoided by fabricating the blades out of several smaller castings, which are joined together afterwards. Furthermore, this modular approach allows the utilisation of different materials in one blade depending on the level of stress applied or corrosion resistance needed in the various regions of one blade.

An established repair technique for high temperature components is transient liquid phase (TLP) bonding. Its application is shown in several studies $[1,2]$. As melting point depressant (MPD) boron or silicon are common, because of their capability of reducing the melting about a suitable amount and diffuse rapidly in nickel-based superalloys. TLP bonding was first reported in the 70ies for nickel-base superalloys by Duvall et al. [3]. This method is based on the diffusion of the MPD elements out of the braze alloy in the parent material to isothermally solidify the molten filler alloy by increasing the melting point. The crystal orientation of the solidified joint will equal the parent material, because the liquid will grow epitaxially at the solid-liquid interface, and the nucleation of new grains in front of the solidification front is suppressed.

There is one problem reported in literature [4-7] regarding boron as MPD: As the solubility of boron in nickel-base alloys is very limited, brittle borides precipitate in the parent alloy around the joint during the brazing process. These precipitations will deteriorate the mechanical properties of the joint [8]. But nevertheless this joining method shows the potential to produce joints with similar mechanical properties as the base material, because the resulting joints are comparable to the base material in regard to composition and microstructure. In literature several studies about bonding different nickel-based superalloys can be found, but the results were never totally satisfying $[5,8]$.

So the intention of the present study is to bond pairings of parent superalloy material with different composition and grain structure, namely one single crystalline and one directionally solidified, by transient liquid phase bonding. A brazing cycle and subsequent heat treatment were developed to obtain a good microstructure regarding the grain boundaries developing and the $\gamma^{\prime}$-amount within the joint. The best process parameters were assessed via the design of experiment (DOE) method. Joints brazed with these brazing parameters were mechanically tested. 


\section{Experimental}

Material. As parent material for the brazing experiments two different nickel-base superalloys were used, one directionally solidified alloy CM247 and one single crystalline alloy PWA1483. The utilised brazing foil MBF80 is commercially available and contains $4 \mathrm{wt} .-\%$ boron as melting point depressant.

Brazing process. The brazing experiments were performed with plate shaped being $10 \times 20 \times 2$ $\mathrm{mm}^{3}$ in size. The $\left(\begin{array}{lll}1 & 0 & 0\end{array}\right)$-orientation of both substrates was perpendicular to the joining surface. The secondary orientations were not controlled. The faying surfaces $\left(10 \times 20 \mathrm{~mm}^{2}\right)$ were polished using 600 grit SiC paper and afterwards ultrasonically cleaned prior to joining. The brazing foil MBF80 was placed between the two prepared surfaces of the substrates and a pressure of approximately 10 $\mathrm{kPa}$ was applied on the parts. Brazing was performed in a high temperature graphite vacuum furnace. The vacuum during heating, melting and brazing was better than $5^{*} 10^{-3} \mathrm{mbar}$. To determine the best brazing parameter for an adequate microstructure experiments were carried out with varying brazing temperature and time.

Characterization. An adequate microstructure was defined as displaying a small number of grain boundaries and a high amount of $\gamma^{\prime}$ in the joint.

To judge the results regarding the grain boundaries the following characteristic number was used: A grid of parallel lines perpendicular to the brazing seam was projected on the joint area and the intersections of these grid lines with grain boundaries were counted. To gain a number independent of the evaluated length of the joint, the intersections were divided by the number of grid lines. This means if the number equals zero, no grain boundary developed in the joint and the value one means one grain boundary or several grains are located in the joint.

The $\gamma^{\prime}$-volume fraction was simply measured as area-fraction in the middle of the joint.

As two process parameters $t$ and $T$ are varied throughout the experiments, they are reduced to one parameter using the Zener-Hollomon-Parameter or "temperature compensated time", which can be calculated according to Eq. 1:

$$
z=t \cdot \exp \left(-\frac{Q}{R T}\right)
$$

where $\mathrm{t}$ is the brazing time, $\mathrm{T}$ the brazing temperature, $\mathrm{Q}$ the activation energy for the boron diffusion in nickel-base superalloys and $\mathrm{R}$ the universal gas constant.

The final assessment of all characteristics together was done via the DOE method, which interpolates the available results over the whole time and temperature range. Based on these results the best brazing parameters were chosen, validated and subsequently applied to generate the samples for the mechanical testing.

Mechanical Testing. The samples for the mechanical tests were brazed with the developed brazing cycle and afterwards the standard heat treatment for PWA1483 was executed to rejuvenate the $\gamma$ '-structure in the joint and the parent material.

To quantify the mechanical properties of these joints, tensile and short term creep rupture tests were performed at room and elevated temperatures to judge the performance at service temperature. These tests were conducted at the Institut für Werkstoffkunde, Technische Universität Darmstadt.

\section{Results}

Microstructure. An overview of the microstructure of a brazed sample can be seen in Fig. 1. The joint hardly shows any porosity and the bonding is very well established, grain boundaries are not present. On the left hand side of the micrograph a detailed view of the particles which precipitated in the SX superalloy PWA1483 adjacent to the joint is displayed. On the right side the same for DS CM247 is shown. In the detailed SEM images the bright white precipitates are carbides, but the needles and small round-shaped precipitates which look a little greyish are borides. In the upper part of the figure a SEM picture of the $\gamma^{\prime}$-structure in the middle of the joint is presented. Here it can clearly be seen that the shape of the $\gamma^{\prime}$-particles is not cuboidal as desired, but rather irregular and the size increased. The same can be observed in the parent material: The shape of the particles is irregular and they are partly solutionized and consequently of an inhomogeneous size distribution. 


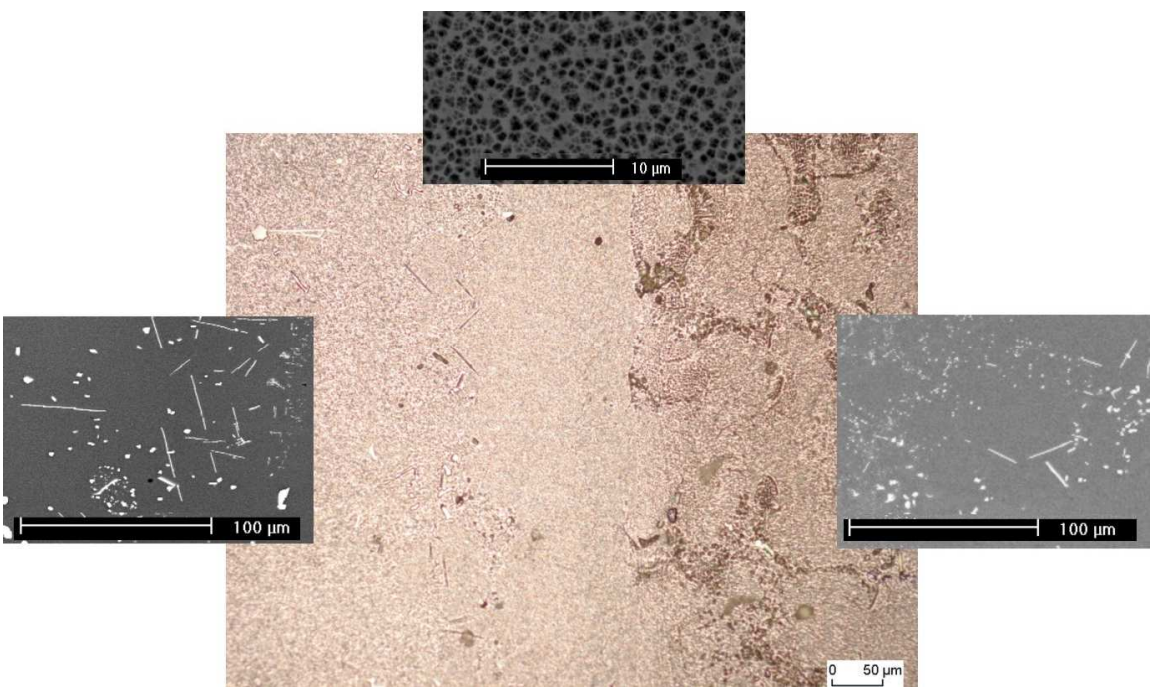

Fig. 1: Overview of a TLP brazing joint: on the left the single crystalline superalloy PWA1483 and a detailed micrograph of the borides, on the right the same for the directionally solidified CM247. On top of the picture the $\gamma^{\prime}$-structure in the middle of the joint can be seen.

The dependence of the $\gamma^{\prime}$-volume fraction on the Zener-Hollomon-Parameter can be seen in Fig. 2. For small parameters the volume fraction of $\gamma^{\prime}$ increases with increasing parameter and converges towards approximately $40-42 \%$ volume fraction for parameter values above 0.0005 .

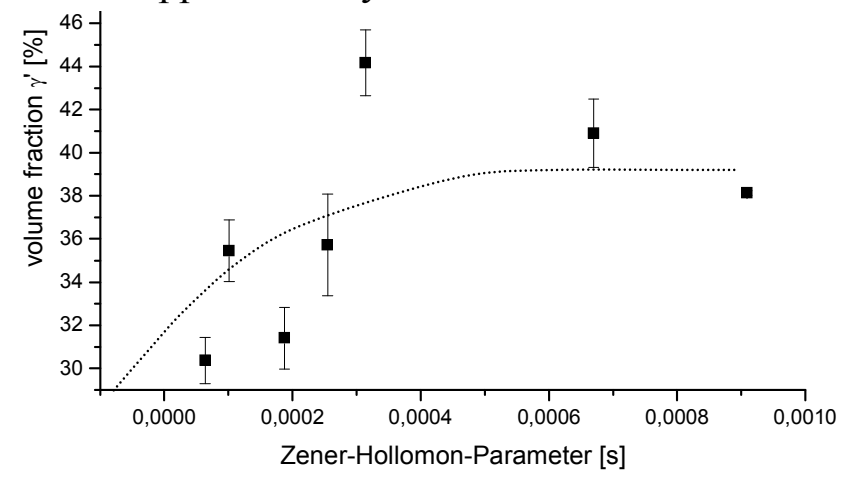

Fig. 2: The $\gamma^{\prime}$-volume fraction as a function of the Zener-Hollomon-Parameter: With increasing temperature compensated time, the $\gamma^{\prime}-$ amount increases and it has reached approximately $40-42 \%$ volume it remains constant.

The amount of $\gamma^{\prime}$ in the joint after the final heat treatment is still slightly, about 2-4\%, smaller than in the surrounding parent material. The element distribution of the $\gamma$ '-forming elements shows in the middle of the joint a slightly lower cumulative content of $\mathrm{Al}$ and $\mathrm{Ti}$ than in the parent materials (Fig. 3).

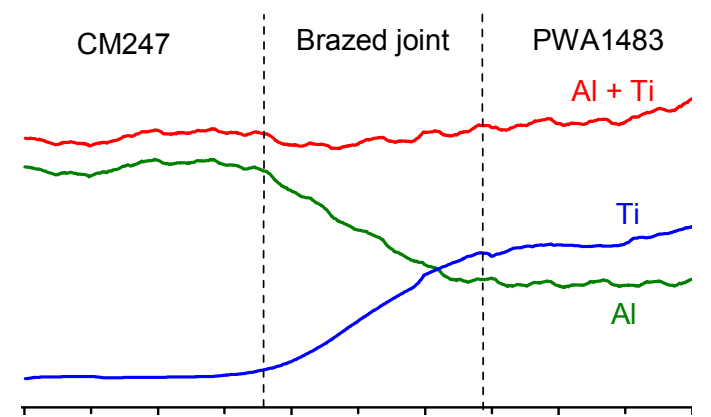

Fig. 3: Element distribution of the $\gamma^{\prime}$-forming elements like aluminium and titanium: the joint contains only a little less $\gamma^{\prime}$-formers than the parent materials as the cumulative curve reveals.

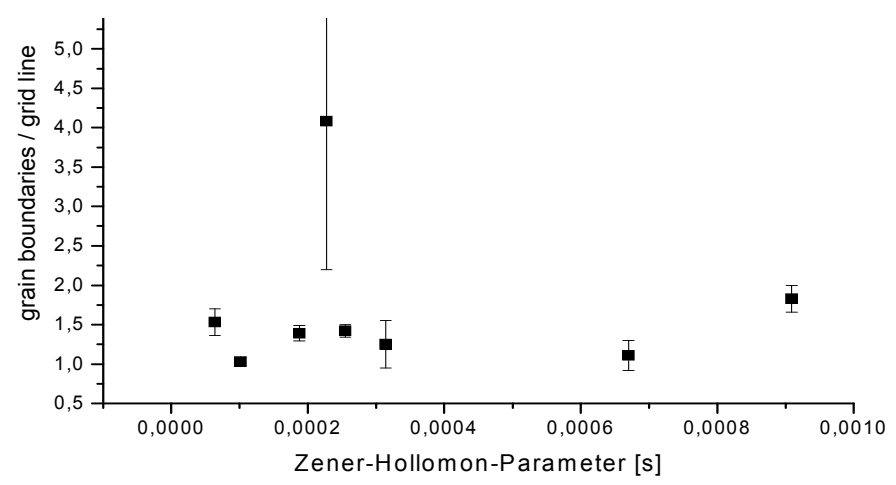

Fig. 4: Grain boundaries divided by the grid lines vs. the Zener-HollomonParameter: All values are within the same range, except one which results by the not completely isothermally solidified joint. 
The grain boundaries which developed in the joint are independent of the brazing parameters (Fig. 4). Only one sample shows an elevated value. This sample did not fully isothermally solidify, instead it contains some remaining eutectic in the middle of the joint. Noticeable is the fact that all of the bonded samples developed at least one grain boundary perpendicular to the solidification direction.

Concerning the boride precipitation in the parent alloys the two superalloys behave differently. Here only the situation after the subsequent heat treatment will be discussed, because this microstructure will determine the mechanical properties: In the CM247 no borides and in the PWA1483 only chinese-script-like borides can be detected.

With the program Minitab ${ }^{\mathrm{TM}}$ the available results were interpolated over the whole time and temperature range via the DOE method. The process parameters for the best achievable microstructure were chosen and the microstructure analysed. The obtained results confirmed the conclusions which were drawn before.

Mechanical Properties. The samples for the mechanical tests were brazed with optimal parameters. To rebuild the $\gamma^{\prime}$-shape in both the joint and the parent material and to further increase the $\gamma^{\prime}-$ volume fraction in the joint after brazing, the standard heat treatment of the superalloy PWA1483 was performed on the test samples.

Mechanical properties such as proof stress and tensile strength are within in the same order of magnitude as the properties of the parent materials (Fig. 5a). At room temperature the brazed sample ruptured in the parent material and shows values of comparable elongation at fracture. It is worth to note, that the brazed samples show significantly lower values of elongation at fracture at $900^{\circ} \mathrm{C}$ and $1000^{\circ} \mathrm{C}$ (Fig. 5a, b). Fracture occurs in the joint and partially in the diffusion affected zone of the adjacent parent material.

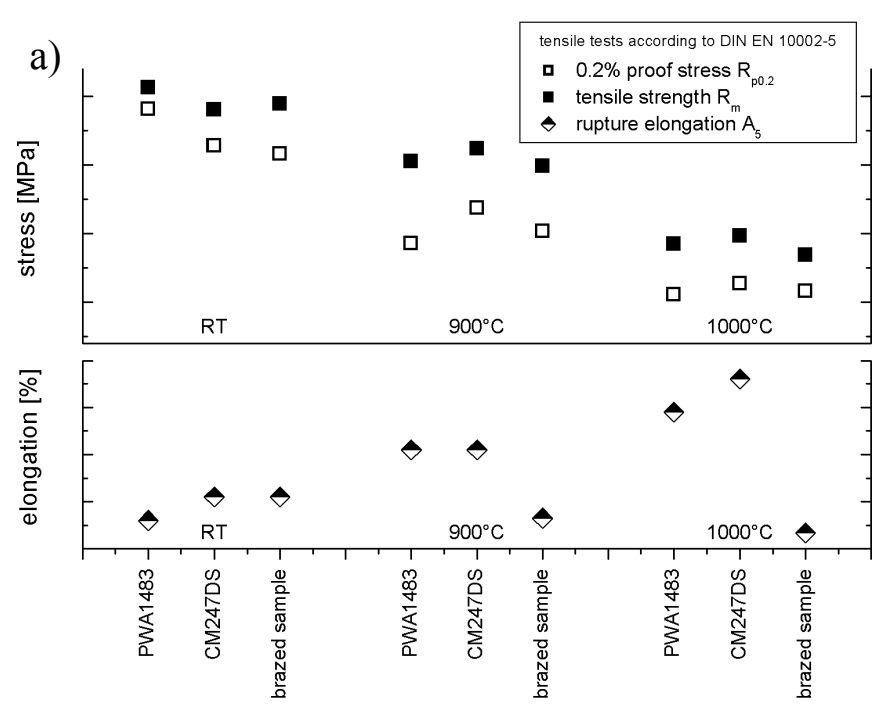

Fig. 5: Tensile properties at room temperature, $900^{\circ} \mathrm{C}$ and $1000^{\circ} \mathrm{C}$; a) proof stress and tensile strength and elongation after fracture; b) stress strain curves at $900^{\circ} \mathrm{C}$; test performed according to DIN EN 10002-5

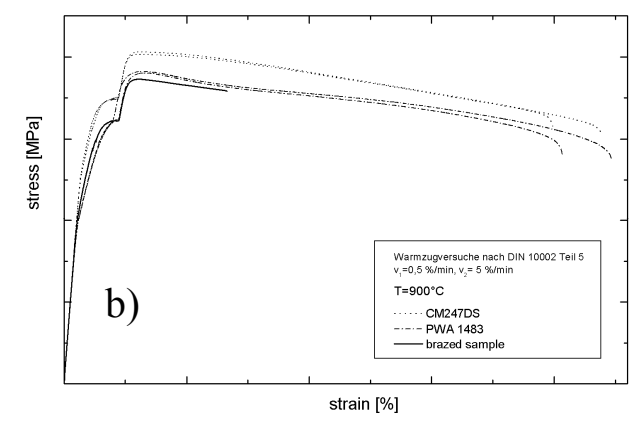

Short-term creep-rupture tests were performed at a temperature of $900^{\circ} \mathrm{C}$ at two distinct stress levels (Fig. 6). They reveal the major drawback of the brazed samples. It is remarkable that the reduction of creep-rupture time by a factor of 1.8 to the weaker parent material is independent of the applied stresses. In terms of stress the reduction amounts to 10\% compared to PWA1483.

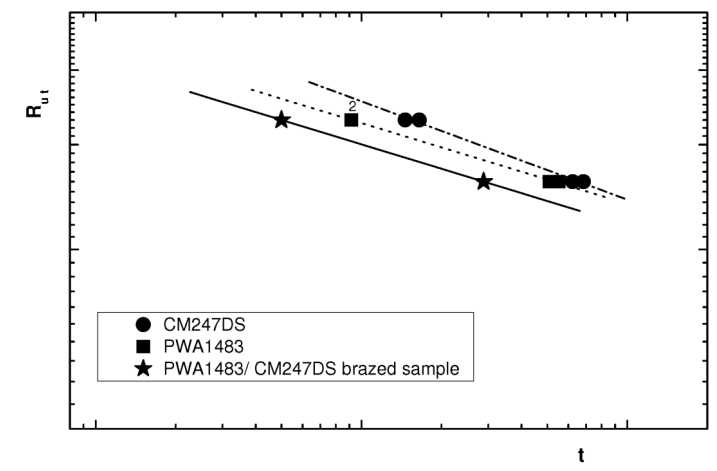

Fig. 6: Results of short term creep rupture tests at $900^{\circ} \mathrm{C}$, tests according to DIN EN ISO 204 


\section{Discussion}

Microstructure. Most descriptions of the transient liquid phase bonding are based on binary systems [9-11]. But the theoretical steps during the process are the same in multicomponent systems. Basically three stages can be distinguished: Widening of the liquid layer, isothermal solidification and homogenization of the bond. The detailed discussion can be found in literature [11].

Brazing time and temperature have a big influence on the $\gamma^{\prime}$-volume fraction in the middle of the joint, as for generation of $\gamma^{\prime}$, aluminium, titanium and/or tantalum is required and the original brazing foil contains none of these elements. The amount of these elements has to increase during the brazing cycle. The $\gamma$ '-forming elements enter the joint volume both during the dissolution of the parent alloys at the beginning of the brazing process and afterwards by diffusional flow. For this special application the dissolution of the parent material plays an important role. Widening depends on the initial boron content in the brazing foil (4 wt.-\%) and it will increase with increasing temperature as the solubility of boron in the base material will decrease. The experiments show for low temperatures a widening of the liquid layer of approximately $100 \%$ and for high temperatures even around $400 \%$. So after the first stage of the TLP bonding process the amount of $\gamma^{\prime}$-formers in the joint will be augmented. It can further be increased by longer brazing times via solid state diffusion of $\mathrm{Al}$, Ti and Ta into the joint. But the influence of the brazing temperature on the $\gamma$ '-volume fraction in the liquid is much more pronounced than that of the brazing time, as solid state diffusion is a slow process.

Regarding the grain boundaries developed in the joint, all samples are similar except one in which developed on average three grains boundaries in the joint. This sample did not fully isothermally solidify and the grains probably developed during cooling of the remaining melt at the end of the brazing cycle, i.e. the specimen was subjected to irregular conditions and it can be neglected. Noticeable is the fact, that all the other samples are in the same range, but all of them developed at least one grain boundary or a few separate grains in the middle of the joint. The most logical explanation [12] of this effect is that the secondary orientations of the two base material plates are twisted against each other. When the liquid starts to solidify, it grows epitaxially on the base materials, but when the two interfaces meet in the middle of the joint the orientations deviate resulting in formation of a grain boundary due to geometrical constraints. This is however process inherent since only by chance the secondary orientation is maintained throughout the joint between DS and SX material.

In this study only the borides which are present after the brazing cycle and the heat treatment are discussed due to their potential impact on mechanical properties. In the directionally solidified alloy CM247 no borides could be detected. The reason for this effect is currently being researched. In the single crystalline alloy PWA1483 the situation is different: Here the alloy shows incipient melting in the interdendritic regions due to the high boron content and following Chinese-script-like borides are formed. They are not precipitated in-situ during the isothermal solidification of the joint as the other forms of borides, but rather during cooling down the part from brazing temperature.

Mechanical Properties. The performed tensile tests show high strength of the brazed samples in the order of magnitude of both parent materials at all test temperatures. It is known from the literature $[1,13]$ that a small joint width contributes to high strength. Furthermore a homogeneous and pore-free microstructure with a minimum of precipitates leads to high strength joints. The joint shows similar microstructure to the base materials. It is assumed that the significant drop in elongation after fracture at high temperatures is due to the lower $\gamma^{\prime}$ content and the change in $\gamma^{\prime}$ morphology which leads to a lower yield strength of the braze [14]. It was shown that after the final heat treatment the $\gamma^{\prime}$-volume fraction in the joint is still about $2-4 \%$ lower. This results in a local deformation of the braze and partially of the adjacent base material.

Time dependent loading in the creep rupture tests lead to a significant reduction in creep rupture life compared to the parent material by a factor of 1.8. The reason for the early failure of the brazed samples is most likely a combination of the lower $\gamma^{\prime}$-amount and $\gamma^{\prime}$-morphology together with the 
grain boundary generated in the middle of the joint, which developed during isothermal solidification. The $\gamma^{\prime}$-phase increases the creep strength via age hardening. A suboptimal $\gamma^{\prime}$-volume fraction and $\gamma^{\prime}$-morphology leads to lower creep strength. Furthermore at grain boundaries the defect density of pores, brittle phases and impurities is increased and this is supposed to contribute to a lower creep performance of the brazed samples [12].

\section{Summary}

In study at hand joints of two distinct superalloys, PWA1483 and CM247, were produced via transient liquid phase bonding. The used braze alloy MBF80 contains boron as melting point depressant. The obtained joints show a homogenous and pore-free microstructure similar to the parent materials. But new grain boundaries developed in the joint and the $\gamma$ '-amount was still lower than in the parent materials. These facts led to high strength of the brazed samples in the performed tensile tests but decrease the elongation at fracture at high temperatures. The creep rupture life was also significantly reduced.

\section{References}

[1] K. Nishimoto, K. Saida, D. Kim and Y. Nakao: ISIJ International Vol. 35 (1995), p. 1298

[2] A. Schnell, A. Stankowske and E. deMarcos: in ASME Turbo Expo, Barcelona, Spain (2006), in press.

[3] D. S. Duvall, W. A. Owczarski and D. F. Paulonis: Welding Journal Vol. 53 (1974), p. 203

[4] A. Schnell: A study of the diffusion brazing process applied to the single crystal superalloy CMSX-4 (Montanuniversität Leoben, Lausanne, Schweiz 2004)

[5] R. Aluru, S. V. Chitti, N. Sofyan, R. D. Love, J. W. Fergus and W. F. Gale: Materials Science and Technology Vol. 24 (2008), p. 517

[6] J. D. Liu, T. Jin, Z. H. Wang, X. F. Sun, H. R. Guan and Z. Q. Hu: Materials Science Forum Vol. 546-549 (2007), p. 1245

[7] M. Pouranvari, A. Ekrami and A. H. Kokabi: Journal of Alloys and Compounds Vol. 461 (2008), p. 341

[8] T. Li, Q. Y. Wang, A. Q. Wang, Z. X. Wen and Z. F. Yue: Key Engineering Materials Vol. 297-300 (2005), p. 1489

[9] I. Tuah-Poku, M. Dollar and T. B. Massalski: Metallurgic Transactions A Vol. 19A (1988), p. 675

[10] W. D. MacDonald and T. W. Eagar: Annual Rev. Materials Science Vol. 22 (1992), p. 23

[11] G. Lesoult: CJM Report (1976)

[12] R. W. Broomfield: in 5th International Charles Parsons Turbine Conference, in press.

[13] O. A. Ojo, N. L. Richards and M. C. Chaturvedi: Science and Technology of Welding and Joining Vol. 9 (2004), p. 209

[14] M. Dinkel, P. Heinz, F. Pyczak, A. Volek, M. Ott, E. Affeldt, A. Vossberg, M. Göken and R. F. Singer: in International Symposium on Superalloys, edited by R. C. Reed, Seven Springs, USA (2008), in press. 